\title{
ANGERMANN, Norbert, Städtisches Leben im Baltikum zur Zeit der Hanse
}

Simonne Abraham-Thisse

\section{OpenEdition}

Journals

Édition électronique

URL : http://journals.openedition.org/ifha/955

DOI : 10.4000/ifha.955

ISSN : 2198-8943

Éditeur

IFRA - Institut franco-allemand (sciences historiques et sociales)

Référence électronique

Simonne Abraham-Thisse, "ANGERMANN, Norbert, Städtisches Leben im Baltikum zur Zeit der Hanse », Revue de l'IFHA [En ligne], Date de recension, mis en ligne le 01 janvier 2004, consulté le 22 septembre 2020. URL : http://journals.openedition.org/ifha/955 ; DOI : https://doi.org/10.4000/ifha.955

Ce document a été généré automatiquement le 22 septembre 2020

(C)IFHA 


\title{
ANGERMANN, Norbert, Städtisches Leben im Baltikum zur Zeit der Hanse
}

\author{
Simonne Abraham-Thisse
}

Le Jubilé des 800 ans en 2001 de la ville de Riga avait déjà suscité de nombreux travaux. On pourra considérer que les onze contributions du 10ème volume du Séminaire Baltique leur apportent un riche complément en se consacrant cette fois à la vie urbaine en Baltique au temps de la Hanse.

Dans son introduction, N.A., l'initiateur du projet, rappelle " pour les non-historiens » (mais cela reste très utile aux historiens!) les épisodes fondamentaux de l'histoire des villes baltiques au temps de la Hanse, délimite les cadres géographiques et chronologiques du thème du séminaire, qu'il complète par quelques observations bien venues sur le rayonnement commercial et sur les rapports entre "Deutsch und Undeutsch » dans ces villes.

À Reval comme à Riga, les frais induits par les fortifications et l'armement représentaient $50 \%$ du montant des dépenses urbaines. À partir des comptes urbains, L. KOTTER (" Die Stadtfestungen Revals », p. 113-124) démontre cependant que le glissement vers l'accroissement des frais d'armement (défense horizontale) aux dépens de ceux des construction (défense verticale) s'est réalisé plus tôt à Riga qu'à Reval dans la seconde moitié du XVe s. En outre, O. SPARIDIS («Städtebau in Livland und Kurland im 16. und 17. Jahrhundert ", p. 125-146) souligne les difficultés que rencontra la ville de Riga, sous l'autorité polonaise puis suédoise, pour concilier dans son évolution architecturale le souci sécuritaire permanent, le pragmatisme de la société marchande et les influences de la Renaissance occidentale. Mais si les ingénieurs suédois planifièrent l'évolution urbaine, ils ne remirent jamais en cause le vieux cœur de ville.

Le développement du commerce en Livonie fut à l'origine de bâtiments affectés spécifiquement aux communautés marchandes. La maison dite des «têtes noires » (en référence à la tête du maure Sankt Mauricius qui ornait leur bannière), réservée aux commissionnaires célibataires, en était une illustration. Mais ce qui retient l'intérêt d'A. FLACHE (« Das Archiv der Rigaer Schwarzenhäupterkompanie », p. 211-230), c'est le devenir des archives que depuis 1441 les frères de cette communauté tentèrent 
jalousement de conserver au travers des vicissitudes de l'histoire. Dispersées en 1940 et maintenant regroupées entre Riga et Marburg, elles constituent encore aujourd'hui un important fonds de recherche, tant pour l'histoire de la ville de Riga que pour celle de l'évolution linguistique allemande.

Depuis la disparition des archives de Riga, les fouilles archéologiques restent la seule source d'information sur la vie quotidienne de la ville médiévale. Elles autorisent A. CAUNE (p. 147-156) à souligner l'absence de ségrégation urbaine entre Allemands et Lettons, qui partageaient les mêmes aliments et dont les élégantes affichaient volontiers, par leurs croix d'ambre ou de bronze, leur religion chrétienne. L'ambiance différait dans les petites villes de Livonie, et l'on saura gré à I. POLSTAM (p. 157-176) de nous avoir dépeint ces villes encore rurales, très dépendantes de l'autorité seigneuriale. Selon l'auteur, l'absence de métiers structurés n'a pas permis l'affirmation d'un patriciat ni celle d'un pouvoir politique urbains. Très présente, l'autorité teutonique aurait maintenu un protectionisme germanique, écartant les non-Allemands (Estoniens et Suédois) de la bourgeoisie, de la propriété et des activités commerciales de la ville. On continuera cependant de s'interroger sur l'origine des fortunes réalisées dans ce contexte par les Estoniens, dont la vie, nous assure l'auteur, trahissait une appréciable aisance.

Il n'en reste pas moins que l'on connait encore mal la structure et le rayonnement de la classe marchande de Riga. En s'appuyant sur les travaux de Dorosenko, M. KOHLER (p. 257-276) nous livre une classification géographique de ces marchands en fonction de leurs axes de commerce. Cependant, leur élite financière, constituée par les "Händler » qui commercent les marchandises venues de Russie, ne participent pas à la vie politique de Riga parce qu'ils sont étrangers, le plus souvent hollandais. C'est à eux en effet que les bourgeois de Riga abandonnent passivement le rôle de commissionnaires et le commerce d'exportation des produits qu'ils ont eux-mêmes importés. Riga ne parvint pas à concilier cette dépendance avec sa volonté de s'opposer à la pénétration de tout capital étranger. Dépourvue du dynamisme de jeunes facteurs qu'aurait pu enrichir une activité internationale, elle contribua à la fermeture et au repli sur soi de sa classe marchande.

I. MISANS (« Die Städte als politischer Faktor in Livland zur Hansezeit », p. 21-42) constate que finalement les villes livoniennes ne sont pas parvenues à constituer une véritable force politique commune et autonome. Certes, elles ont progressivement cessé de s'identifier à la Hanse. Mais ce fut sans doute pour se rendre davantage dépendantes des autorités seigneuriales, qu'elles fussent rurales, teutoniques ou religieuses.

Pour sa part, Th. BRUCK (p. 43-92) retrace plus particulièrement les tentatives d'émancipation nationale, politique et économique de Riga aux XIVe et XVe s. L'auteur souligne fort justement les liens de cause à effet existant entre la détermination de Riga à pénétrer les marchés lithuaniens et russes par l'intermédiaire de sa Grande Guilde des marchands et sa volonté d'affirmer son identité livonienne face aux Allemands, cela malgré la vive résistance de Lübeck, inquiète pour les privilèges de ses Novgorodfahrer. Orientation commerciale et indépendance nationale vont donc de pair à Riga. Mais, selon l'auteur, malgré l'appui que Riga trouva auprès des villes prussiennes, qui partageaient sa résistance à l'hégémonie lübeckoise et lui servaient d'intermédiaire commercial avec l'Occident, les résultats des tentatives restèrent inférieurs aux espérances. Et Riga n'atteignit jamais non plus l'expansion commerciale de Reval, plus 
souple dans ses relations avec Lübeck. J. KHREM (" Stadt und Landesherr als Geschäftspartner. Die Handelsgelegenheiten in den Beziehungen zwischen dem deutschen Orden und der Stadt Reval ", p. 93-112) souligne en effet les liens commerciaux et financiers qui subsistèrent entre marchands de Reval et marchands de l'Ordre jusqu'au milieu des conflits du XVe s.

L'exécution de deux Russes à Reval, l'un pour sodomie, l'autre pour faux monnayage, fut-elle la cause ou le prétexte de la fermeture du comptoir de Novgorod en 1494 et du long emprisonnement des Hanséates qui s'ensuivit ? Parmi les historiens qui se posent la question, A. SELART ( Z Zur Geschichte der Russen in Livland um die Wende des 15. zum 16. Jahrhundert. Der Vorwand zur Schließung des St. Peterhofes in Novgorod im Jahr 1494 », p. 177-210) tente ici de sonder les événements annonciateurs (annexion du comptoir par Ivan III en 1478, déportation des populations de Novgorod, confiscation des biens, immigration de nouveaux Russes, guerre russo-livonienne, spoliation supposée des envoyés grecs du prince de Moscou, à Reval) et s'attache à décrypter l'image que les chrétiens occidentaux se faisaient des Russes selon eux schismatiques. L'auteur reprend, en les comparant, les récits des différentes chroniques hanséatiques, livoniennes et russes, qui se font l'écho de positions aussi convaincues que contradictoires sur ladite exécution. Il met en évidence le fait qu'au-delà de la volonté politique d'Ivan III d'imposer l'ordre moscovite dans ses territoires, se dresse une véritable confrontation juridique, culturelle et religieuse des deux communautés germano-livonienne et russe.

En s'appuyant sur les études de S. Jenks, de W. Stark et de W. Paravicini, Kl. MILITZER (" Der Handel der livländischen Kaufleute mit Brügge und London », p. 231-256) en arrive, malgré l'imprécision des sources, à conclure que les Livoniens, au contraire des Vendes et des Prussiens, se sont peu livrés à un commerce direct avec l' Angleterre au Moyen Âge. Si les marchands de Reval s'attachaient davantage à un commerce direct avec Bruges, ceux de Riga auraient préféré recourir à l'intermédiaire lübeckois pour effectuer leur commerce occidental.

Bien que l'on eût souhaité y trouver des plans (notamment de Reval et de Riga) et des cartes plus explicites (pour les petites villes), ce volume présente finalement un intérêt multiple. Il souligne d'abord à la fois l'originalité des villes livoniennes et leurs nuances particulières : on ne vit pas à Neu Pernau comme à Reval ou Riga. En outre, si les activités économiques et commerciales ne sont que peu abordées, elles sont toujours présentes en filigrane comme élément déterminant de l'évolution de ces villes. On sent cependant que trois thèmes demeurent très sensibles : la confrontation des Allemands et des non-Allemands (dans les villes livoniennes comme à Novgorod), le poids des dépendances (vis-à-vis de la Hanse, des féodaux, des Polonais et des Suédois), mais surtout, et c'est la conclusion globale de l'ouvrage, l'impossible ascension politique d'un patriciat urbain en Livonie au Moyen- Âge.

Simonne ABRAHAM-THISSE (Centre National de la Recherche

Scientifique) 